RUNNING TITLE: BMI polygenic score, early stress, and depression.

\title{
A Polygenic Score for Body Mass Index is Associated with Depressive Symptoms via Early Life Stress: Evidence for gene- environment correlation
}

\author{
Reut Avinun and Ahmad R. Hariri
}

Laboratory of NeuroGenetics, Department of Psychology \& Neuroscience, Duke University, Durham, NC, USA

\section{FUNDING AND DISCLOSURE}

We would like to thank the participants of the Duke Neurogenetics Study and members of the Laboratory of NeuroGenetics, especially Annchen R. Knodt, Spenser R. Radtke, and Bartholomew D. Brigidi for their assistance with data collection and analysis. The DNS was supported by Duke University as well as US-National Institutes of Health grants R01DA033369 and R01DA031579. RA and ARH received further support from US-National Institutes of Health grant R01AG049789. The Brain Imaging and Analysis Center was supported by the Office of the Director, National Institutes of Health under Award Number S100D021480. The authors declare no competing financial or other interests.

Corresponding Author: Reut Avinun, Ph.D., Laboratory of NeuroGenetics, Department of Psychology \& Neuroscience, Duke University, Grey Building 2020 West Main St, Ste 0030 Durham, NC 27705. Email: reut.avinun@duke.edu 


\section{$1 \quad$ ABSTRACT}

2 Background: Increasing childhood overweight and obesity rates are associated with

3 not only adverse physical, but also mental health outcomes, including depression.

4 These negative outcomes may be caused and/or exacerbated by the bullying and

5 shaming overweight individuals experience. As body mass index (BMI) can be highly

6 heritable, we hypothesized that a genetic risk toward higher BMI, will predict higher

7 early life stress (ELS), which in turn will predict higher depressive symptoms in

8 adulthood. Such a process will reflect an evocative gene-environment correlation

9 ( $\mathrm{rGE}$ ) wherein an individual's genetically influenced phenotype evokes a reaction

10 from the environment that subsequently shapes the individual's health.

11 Methods: We modeled genetic risk using a polygenic score of BMI derived from a recent large GWAS meta-analysis. Self-reports were used for the assessment of ELS and depressive symptoms in adulthood. The discovery sample consisted of 524 nonHispanic Caucasian university students from the Duke Neurogenetics Study (DNS; 278 women, mean age $19.78 \pm 1.23$ years) and the independent replication sample consisted of 5930 white British individuals from the UK biobank (UKB; 3128 women, mean age $62.66 \pm 7.38$ years).

Results: A significant mediation effect was found in the DNS (indirect effect=.207, bootstrapped $\mathrm{SE}=.10,95 \% \mathrm{Cl} .014$ to .421 ), and then replicated in the UKB (indirect effect $=.04$, bootstrapped $\mathrm{SE}=.01,95 \% \mathrm{Cl}: .018$ to .066$)$. Higher $\mathrm{BMI}$ polygenic scores were associated with higher depressive symptoms through the experience of higher 
bioRxiv preprint doi: https://doi.org/10.1101/536938; this version posted January 31, 2019. The copyright holder for this preprint (which was not certified by peer review) is the author/funder, who has granted bioRxiv a license to display the preprint in perpetuity. It is made available under aCC-BY-NC-ND 4.0 International license.

23 Conclusions: Our findings suggest that evocative rGE may contribute to weight-

24 related mental health problems and stress the need for interventions that aim to

25 reduce weight bias, specifically during childhood. 
Overweight individuals suffer from stigmatization, bias, and bullying, from multiple sources including peers, health care providers, educators, and, most surprisingly perhaps, family members [1]. In a study of adolescents enrolled in weight loss camps, 37\% reported being teased or bullied by a parent [2]. Another study on 2449 women recruited from a weight loss support group organization, found that $44 \%$ experienced stigma from their mothers more than once, while $34 \%$ experienced it from their fathers [3]. As weight-related teasing has been shown to predict depression and lower self-esteem [4, 5], it may represent another form of early life stress (ELS) that is associated with various negative physical and mental health outcomes $[6,7]$.

Gene environment correlations [ $\mathrm{rGE} ; 8,9]$ can represent passive, evocative, and active processes that create associations between individuals' genes and the environment. Evocative rGE, which refers to instances in which a genetically influenced phenotype of an individual evokes a certain reaction from the environment, may be relevant to weight-related teasing and bullying, so that individuals with a genetic propensity toward a higher body mass index (BMI), will be more likely to experience teasing, especially in the current Western cultural climate, which is characterized by negative and prejudicial attitudes towards overweight and obese individuals [10].

A recent meta-analysis of genome-wide association studies (GWAS; [11]), consisting of 681275 participants on average, explained 5\% of the variance in BMI with GWAS significant single nucleotide polymorphisms (SNPs). In the current study, we hypothesized that a polygenic score based on the results from this meta-analysis, will predict early life stress (ELS), consistent with evocative rGE, which in turn will 
predict depressive symptoms in adulthood. We tested our hypothesis in two independent samples: a discovery sample of 524 non-Hispanic Caucasian university students from the Duke Neurogenetics Study and a replication sample of 5930 adult white British volunteers from the UK Biobank (UKB). Although the GWAS metaanalysis included data from the UKB , current BMI was not a phenotype of interest in our study, and therefore the overlap should not bias our analyses. Nonetheless, to validate our results in the analyses that included UKB data, we also used BMI polygenic scores that were based on a GWAS that did not include the UKB as a discovery sample [12].

\section{MATERIALS AND METHODS}

\section{Participants}

Our discovery sample consisted of 524 self-reported non-Hispanic Caucasian participants (278 women, mean age $19.78 \pm 1.23$ years) from the Duke Neurogenetics Study (DNS) for whom there was complete data on genotypes, ELS, depressive symptoms, and all covariates. All procedures were approved by the Institutional Review Board of the Duke University Medical Center, and participants provided informed consent before study initiation. All participants were free of the following study exclusions: 1) medical diagnoses of cancer, stroke, diabetes requiring insulin treatment, chronic kidney or liver disease, or lifetime history of psychotic symptoms; 2) use of psychotropic, glucocorticoid, or hypolipidemic medication; and 3) conditions affecting cerebral blood flow and metabolism (e.g., hypertension). Importantly, neither current nor lifetime diagnosis were an exclusion criterion, as the 
DNS sought to establish broad variability in multiple behavioral phenotypes related to psychopathology.

The replication sample consisted of 5930 white British individuals (3 128 women, mean age $62.66 \pm 7.38$ years), who participated in the UKB's imaging wave, completed an online mental health questionnaire [13], and had complete genotype, ELS, depressive symptoms and covariate data. The UKB [www.ukbiobank.ac.uk; 14] includes over 500,000 participants, between the ages of 40 and 69 years, who were recruited within the UK between 2006 and 2010. The UKB study has been approved by the National Health Service Research Ethics Service (reference: 11/NW/0382), and our analyses were conducted under UKB application 28174.

\section{Race/Ethnicity}

Because self-reported race and ethnicity are not always an accurate reflection of genetic ancestry, an analysis of identity by state of whole-genome SNPs in the DNS was performed in PLINK [15]. The first two multidimensional scaling components within the non-Hispanic Caucasian subgroup were used as covariates in analyses of data from the DNS. The decision to use only the first two components was based on an examination of a scree plot of the variance explained by each component. For analyses of data from the UKB, only those who were 'white British' based on both self-identification and a principal components analysis of genetic ancestry were included. Additionally, the first 10 multidimensional scaling components received from the UKB's data repository (unique data identifiers: 22009-0.1-22009-0.10) were included as covariates as previously done [e.g., 16]. 
Body Mass Index (BMI)

In both DNS and UKB samples, BMI was calculated at the time of imaging based on the height and weight of the participants. In the DNS, this calculation was based on imperial system values (pounds/inches2*703), while in the UKB the metric system was used $(\mathrm{kg} / \mathrm{m} 2)$. In the DNS $1.3 \%$ of the sample was obese, compared to $18.7 \%$ in the UKB.

\section{Depressive symptoms}

In the DNS, the 20-item Center for Epidemiologic Studies Depression Scale (CES-D) was used to asses depressive symptoms in the past week [17]. All items were summed to create a total depressive symptoms score. In the UKB, the Patient Health Questionnaire 9-question version (PHQ-9) was used to asses depressive symptoms in the past 2 weeks [18]. All items were summed to create a total depressive symptoms score.

\section{Early life stress}

In the DNS, ELS was estimated using the Childhood Trauma Questionnaire [CTQ; 19].

The CTQ has 28-items and it assesses the frequency of emotional, physical, and sexual abuse as well as emotional and physical neglect. The scores on the 5 subscales (each ranging from 5 to 25 ) were summed to create a total score of ELS. In the UKB, the Childhood Trauma Screener -5 item (CTS-5) was used to assess adverse events during childhood [20]. CTS-5 is a short version of the CTQ consisting of 5 items: "Felt hated by family member as a child", "Physically abused by family as a child", "Felt loved as a child" (reverse coded), "Sexually molested as a child", and 
121

"Someone to take to doctor when needed as a child" (reverse coded). The 5 items, each ranging from $0-4$, were summed to create a total score of ELS.

\section{Genotyping}

In the DNS, DNA was isolated from saliva using Oragene DNA self-collection kits (DNA Genotek) customized for 23andMe (www.23andme.com). DNA extraction and genotyping were performed through 23andMe by the National Genetics Institute (NGI), a CLIA-certified clinical laboratory and subsidiary of Laboratory Corporation of America. One of two different Illumina arrays with custom content was used to provide genome-wide SNP data, the HumanOmniExpress $(\mathrm{N}=329)$ or HumanOmniExpress-24 [N=195;21, 22, 23]. In the UKB, samples were genotyped using either the UK BiLEVE $(\mathrm{N}=569)$ or the UKB axiom $(\mathrm{N}=5,361)$ array. Details regarding the UKB's quality control can be found elsewhere[24].

\section{Quality control and polygenic scoring}

For genetic data from both the DNS and UK Bionbank, PLINK v1.90 [15] was used to apply quality control cutoffs and exclude SNPs or individuals based on the following criteria: missing genotype rate per individual >.10, missing rate per SNP >.10, minor allele frequency $<.01$, and Hardy-Weinberg equilibrium $p<1 e-6$. Additionally, in the UKB, quality control variables that were provided with the dataset were used to exclude participants based on a sex mismatch (genetic sex different from reported sex), a genetic relationship to another participant, outliers for heterozygosity or missingness (unique Data Identifier 22010-0.0), and UKBiLEVE genotype quality control for samples (unique Data Identifiers 22050-0.0-22052-0.0). 
Polygenic scores were calculated using PLINK's [15] "--score" command based on published SNP-level summary statistics from a recent BMI GWAS meta-analysis [11]. SNPs from the GWAS of BMI meta-analysis were matched with SNPs from the DNS and the UKB. For each SNP the number of the alleles $(0,1$, or 2$)$ associated with BMI was multiplied by the effect estimated in the GWAS. The polygenic score for each individual was an average of weighted BMI-associated alleles. All SNPs matched with SNPs from the DNS and UKB were used regardless of effect size and significance in the original GWAS, as previously recommended and shown to be effective [25, 26]. A total of 442040 SNPs from the DNS and 648530 SNPs from the UKB were included in the polygenic scores. The approach described here for the calculation of the polygenic scores was successfully used in previous studies [e.g., 27, 28-30]. For validation of the indirect effect in the UKB, BMI polygenic scores were also calculated based on an older GWAS that did not include the UKB as a discovery sample [12].

\section{Statistical analysis}

Linear regression analyses in SPSS v25 were conducted to test for an association between the BMI polygenic score and BMI in adulthood. The PROCESS SPSS macro,

162 version 3.1 [31], was used to conduct the mediation analyses. Participants' sex 163 (coded as $0=$ =males, $1=$ females), age, and ethnicity genomic components were 164 entered as covariates in all analyses. In the mediation analyses, bias-corrected 165 bootstrapping (set to 5,000) was used to allow for non-symmetric $95 \%$ confidence 166 intervals (Cls). Specifically, indirect effects are likely to have a non-normal 167 distribution, and consequently the use of non-symmetric Cls for the determination of 168 significance is recommended [32]. However, bias-corrected bootstrapping also has 
169

170

171

172

173

174

175

its faults [33] and, consequently, as supportive evidence for the indirect effect, we also present the test of joint significance, which examines whether the a path (BMI polygenic score to ELS) and the $b$ path (ELS to depressive symptoms, while controlling for the BMI polygenic score) are significant. The BMI polygenic scores were standardized to make interpretability easier. The mediation was first analyzed in the DNS, and then a replication was tested in the UKB. As a validation of the indirect effect in the UKB, it was also tested with an older BMI polygenic score that was not based on a GWAS that included the UKB [12].

\section{RESULTS}

Descriptive statistics are presented in table 1.

\section{Confirming an association between BMI polygenic scores and measured BMI}

As a preliminary analysis we confirmed that higher BMI polygenic scores were significantly associated with higher measured BMI in both the DNS ( $N=522, b=.837$, $\mathrm{SE}=.117, \mathrm{p}<.001)$ and the UKB $(\mathrm{N}=5925, \mathrm{~b}=1.41, \mathrm{SE}=.054, \mathrm{p}<.001)$. (notably, the UKB was included in the BMI GWAS, and consequently the significant association is expected and possibly somewhat inflated). These associations were robust to the inclusion of sex, age, and ethnicity genomic components as covariates. The sample sizes for these analyses were slightly different from the mediation analyses below because measured BMI was missing for a few participants. 
193

The BMI polygenic scores were significantly associated with ELS $(b=.65, S E=.31$, $p=.038$ ), so that higher scores predicted higher ELS. Of the covariates, age was significantly and negatively associated with $\operatorname{ELS}(b=-.73, \mathrm{SE}=.25, p<.01)$.

ELS predicts depressive symptoms (b path) in the DNS

With the BMI polygenic scores in the model, ELS significantly and positively predicted depressive symptoms $(b=.32, \mathrm{SE}=.04, \mathrm{p}<.001)$.

\section{$B M I$ polygenic scores predict depressive symptoms in the DNS}

The BMI polygenic scores did not significantly predict depressive symptoms $(b=-.34$,

$\mathrm{SE}=.31$, ns). Notably, however, the significance of a direct path from $\times$ (BMI polygenic scores) to $\mathrm{Y}$ (depressive symptoms) or the 'total effect' (the ' $c$ ' path), is not a prerequisite for the testing of a mediation/indirect effect [34-36].

\section{Mediation model in the DNS}

The indirect path $\left(a^{*} b\right), \mathrm{BMI}$ polygenic scores to ELS to depressive symptoms was significant as indicated by the bias corrected bootstrapped $95 \% \mathrm{Cl}$ not including zero (Figure 1a; indirect effect=.207, bootstrapped SE=.10, 95\% Cl: .014 to .421).

\section{Mediation Model in the UBK}

The a path, from the BMI polygenic scores to ELS, and the $b$ path, from ELS to depressive symptoms while controlling for BMI polygenic scores, were significant (a path: $\mathrm{b}=.10, \mathrm{SE}=.03, \mathrm{p}<.01 ; b$ path: $\mathrm{b}=.40, \mathrm{SE}=.02, \mathrm{p}<.001)$. The indirect path also replicated (Figure $1 \mathrm{~b}$; indirect effect=.04, bootstrapped $\mathrm{SE}=.01,95 \% \mathrm{Cl}$ : .018 to .066), 
supporting a mediation in which BMI polygenic scores are associated with depressive symptoms indirectly through ELS. Similar results were obtained with the BMI polygenic scores that were based on a GWAS that did not include the UKB as a discovery sample (indirect effect=.026, bootstrapped $\mathrm{SE}=.01,95 \% \mathrm{Cl} . .004$ to .05 ).

\section{DISCUSSION}

Here, in two independent samples, we provide novel evidence supporting evocative rGE as a possible mechanism in weight-related depression. We demonstrate a significant mediation in which higher GWAS-derived BMI polygenic scores are associated with higher levels of depressive symptoms in adulthood through elevated levels of ELS. These results suggest that in the current Western cultural climate, having a genetic makeup that increases the risk of a high BMI, may lead to a phenotype that evokes increased stress, which increases the experience of depressive symptoms in adulthood.

Various studies have reported links between being overweight and experiencing stigmatization, teasing, and bullying from peers, educators, co-workers, health care providers, and family members [1]. This negativity can lead to adverse mental health outcomes, including depression [5], but is not limited to mental health. Obesity, childhood trauma, and depression have all been linked to physical illness including cardiovascular disease, type 2 diabetes, and autoimmune disorders $[6,37-40]$

While several strategies have been proposed to battle the growing prevalence of childhood obesity, including nutrition standards for school meals; improved early care and education; and increased access to adolescent bariatric surgery [41], our 
findings further encourage weight stigma reduction efforts, specifically among family members and parents. In addition to the myriad of mental and physical health disorders that are associated with ELS and childhood trauma, one of the most prevalent coping responses to weight stigma is eating [3]. Consequently, ELS may 245 lead to additional weight gain and is itself a risk factor for obesity [42]. Thus, interventions that aim to reduce weight stigma may have a broad positive effect on 247 health.

Although our study has several strengths, including the use of two independent samples with markedly different characteristics (e.g., young university students versus older community volunteers) and a GWAS-derived polygenic score, it is not without limitations. First, retrospective reports were used for the estimation of ELS and childhood trauma. Ideally, prospective data should be used to model ELS in the absence of reporting bias. Second, we did not have measures of childhood BMI in either sample. Although previous research does support a link between childhood $\mathrm{BMI}$, teasing, and depression, and genetic influences on BMI have been shown to be 256 relatively stable throughout development [43, 44], genetically informed longitudinal studies across development are needed to further validate our findings. Third, the 258 non-Hispanic Caucasian DNS sample is relatively homogeneous in terms of social background, which may have led to an underestimation of the effect in this sample.

260 Fourth, our findings are limited to populations of European descent and to the 261 Western culture. Additional research in diverse populations is needed to determine 262 the extent to which the observed evocative rGE mechanism shapes weight-related mental health. Further replication is also needed to evaluate the potential of the BMI polygenic score as a risk biomarker of depression associated with ELS. 
265

266

267

268

269

270

271

272

273

274

275

276

277

278

279

280

281

283

284

285

\section{References}

1. Puhl RM, Latner JD. Stigma, obesity, and the health of the nation's children. Psychol. Bull. 2007; 133(4): 557.

2. Puhl RM, Peterson JL, Luedicke J. Weight-based victimization: Bullying experiences of weight loss treatment-seeking youth. Pediatrics 2013; 131(1): e1-e9.

3. Puhl RM, Brownell KD. Confronting and coping with weight stigma: an investigation of overweight and obese adults. Obesity 2006; 14(10): 18021815.

4. Greenleaf C, Petrie TA, Martin SB. Relationship of weight-based teasing and adolescents' psychological well-being and physical health. J. Sch. Health 2014; 84(1): 49-55.

5. Greenleaf C, Petrie T, Martin S. Exploring weight-related teasing and depression among overweight and obese adolescents. Revue Européenne de Psychologie Appliquée/European Review of Applied Psychology 2017; 67(3): 147-153. 
6. Goodwin RD, Stein MB. Association between childhood trauma and physical disorders among adults in the United States. Psychol. Med. 2004; 34(3): 509520.

7. Mersky JP, Topitzes J, Reynolds AJ. Impacts of adverse childhood experiences on health, mental health, and substance use in early adulthood: A cohort study of an urban, minority sample in the US. Child Abuse Negl. 2013; 37(11): 917-925.

8. Plomin R, DeFries JC, Loehlin JC. Genotype-environment interaction and correlation in the analysis of human behavior. Psychol. Bull. 1977; 84(2): 309-

9. Avinun R, Knafo-Noam A. Socialization, Genetics and their Interplay in Development. In: Grusec JE, Hastings PD (eds). Handbook of Socialization: Theory and research, 2 edn. Guilford Press, 2014, pp 347-371.

10. Pearl RL. Weight bias and stigma: public health implications and structural 
11. Yengo L, Sidorenko J, Kemper KE, Zheng Z, Wood AR, Weedon MN et al. Meta-analysis of genome-wide association studies for height and body mass index in 700,000 individuals of European ancestry. bioRxiv 2018: 274654.

12. Locke AE, Kahali B, Berndt SI, Justice AE, Pers TH, Day FR et al. Genetic studies of body mass index yield new insights for obesity biology. Nature 2015; 518(7538): 197.

13. Davis KA, Coleman JR, Adams M, Allen N, Breen G, Cullen B et al. Mental health in UK Biobank: development, implementation and results from an online questionnaire completed by 157366 participants. BJPsych Open 2018; 4(3): 83-90.

14. Sudlow C, Gallacher J, Allen N, Beral V, Burton P, Danesh J et al. UK biobank: an open access resource for identifying the causes of a wide range of complex diseases of middle and old age. PLoS Med. 2015; 12(3): e1001779.

15. Purcell S, Neale B, Todd-Brown K, Thomas L, Ferreira MA, Bender D et al. PLINK: a tool set for whole-genome association and population-based linkage analyses. The American Journal of Human Genetics 2007; 81(3): 559-575. 
16. Whalley HC, Adams MJ, Hall L, Clarke T-K, Fernandez-Pujals AM, Gibson J et al. Dissection of major depressive disorder using polygenic risk scores for schizophrenia in two independent cohorts. Translational Psychiatry 2016; 6(11): e938.

17. Radloff LS. The CES-D scale: A self-report depression scale for research in the general population. Applied psychological measurement 1977; 1(3): 385-401.

18. Kroenke K, Spitzer RL, Williams JB. The PHQ-9: validity of a brief depression severity measure. J. Gen. Intern. Med. 2001; 16(9): 606-613.

19. Bernstein DP, Stein JA, Newcomb MD, Walker E, Pogge D, Ahluvalia T et al. Development and validation of a brief screening version of the Childhood Trauma Questionnaire. Child Abuse Negl. 2003; 27(2): 169-190.

20. Glaesmer H, Schulz A, Häuser W, Freyberger HJ, Brähler E, Grabe H-J. The childhood trauma screener (CTS)-development and validation of cut-offscores for classificatory diagnostics. Psychiatr. Prax. 2013; 40(4): 220-226.

21. Do CB, Tung JY, Dorfman E, Kiefer AK, Drabant EM, Francke U et al. Webbased genome-wide association study identifies two novel loci and a 
substantial genetic component for Parkinson's disease. PLoS Genet. 2011; 7(6): e1002141.

22. Eriksson N, Macpherson JM, Tung JY, Hon LS, Naughton B, Saxonov S et al. Web-based, participant-driven studies yield novel genetic associations for common traits. PLoS Genet. 2010; 6(6): e1000993.

23. Tung JY, Do CB, Hinds DA, Kiefer AK, Macpherson JM, Chowdry AB et al. Efficient replication of over 180 genetic associations with self-reported medical data. PLOS ONE 2011; 6(8): e23473.

24. Bycroft C, Freeman C, Petkova D, Band G, Elliott LT, Sharp K et al. Genomewide genetic data on 500,000 UK Biobank participants. bioRxiv 2017: 166298.

25. Dudbridge F. Power and predictive accuracy of polygenic risk scores. PLoS Genet. 2013; 9(3): e1003348.

26. Ware EB, Schmitz LL, Faul JD, Gard A, Mitchell C, Smith JA et al. Heterogeneity 
27. Domingue BW, Belsky DW, Conley D, Harris KM, Boardman JD. Polygenic influence on educational attainment: New evidence from the National Longitudinal Study of Adolescent to Adult Health. AERA open 2015; 1(3): 2332858415599972.

28. Domingue BW, Liu H, Okbay A, Belsky DW. Genetic heterogeneity in depressive symptoms following the death of a spouse: Polygenic score analysis of the US Health and Retirement Study. Am. J. Psychiatry 2017; 174(10): 963-970.

29. Stephan $Y$, Sutin AR, Luchetti M, Caille P, Terracciano A. Polygenic Score for Alzheimer Disease and cognition: The mediating role of personality. J. Psychiatr. Res. 2018.

30. Marioni RE, Ritchie SJ, Joshi PK, Hagenaars SP, Okbay A, Fischer $\mathrm{K}$ et al. Genetic variants linked to education predict longevity. Proc. Natl. Acad. Sci. U. S. A. 2016; 113(47): 13366-13371.

31. Hayes AF. Introduction to mediation, moderation, and conditional process analysis: A regression-based approach, Guilford Publications, 2017. 
390

32. Mackinnon DP, Lockwood CM, Williams J. Confidence Limits for the Indirect Effect: Distribution of the Product and Resampling Methods. Multivariate Behavioral Research 2004; 39(1): 99.

33. Hayes AF, Scharkow M. The relative trustworthiness of inferential tests of the indirect effect in statistical mediation analysis: Does method really matter? Psychol. Sci. 2013; 24(10): 1918-1927.

34. MacKinnon DP, Krull JL, Lockwood CM. Equivalence of the mediation, confounding and suppression effect. Prev. Sci. 2000; 1(4): 173-181.

35. Rucker DD, Preacher KJ, Tormala ZL, Petty RE. Mediation analysis in social psychology: Current practices and new recommendations. Social and Personality Psychology Compass 2011; 5(6): 359-371.

36. Hayes AF. Beyond Baron and Kenny: Statistical mediation analysis in the new millennium. Communication monographs 2009; 76(4): 408-420.

37. Carney RM, Freedland KE. Depression and coronary heart disease. Nature Reviews Cardiology 2017; 14(3): 145. 
38. Versini $M$, Jeandel $P-Y$, Rosenthal $E$, Shoenfeld $Y$. Obesity in autoimmune diseases: not a passive bystander. Autoimmun. Rev. 2014; 13(9): 981-1000.

39. Kahn SE, Hull RL, Utzschneider KM. Mechanisms linking obesity to insulin resistance and type 2 diabetes. Nature 2006; 444(7121): 840.

40. Van Gaal LF, Mertens IL, Christophe E. Mechanisms linking obesity with cardiovascular disease. Nature 2006; 444(7121): 875.

41. Gortmaker SL, Wang YC, Long MW, Giles CM, Ward ZJ, Barrett JL et al. Three interventions that reduce childhood obesity are projected to save more than they cost to implement. Health Aff. (Millwood) 2015; 34(11): 1932-1939.

42. Danese A, Tan M. Childhood maltreatment and obesity: systematic review and meta-analysis. Mol. Psychiatry 2014; 19(5): 544.

43. Haberstick BC, Lessem JM, McQueen MB, Boardman JD, Hopfer CJ, Smolen A et al. Stable genes and changing environments: body mass index across adolescence and young adulthood. Behav. Genet. 2010; 40(4): 495-504. 
bioRxiv preprint doi: https://doi.org/10.1101/536938; this version posted January 31, 2019. The copyright holder for this preprint (which was not certified by peer review) is the author/funder, who has granted bioRxiv a license to display the preprint in perpetuity. It is made available under aCC-BY-NC-ND 4.0 International license.

431 44. Silventoinen K, Kaprio J. Genetics of tracking of body mass index from birth to late middle age: evidence from twin and family studies. Obes. Facts 2009;

433 2(3): 196-202.

434

435

436

437 
Table 1. Descriptive statistics of study variables.

\begin{tabular}{lcccccccc} 
& \multicolumn{3}{c}{ DNS } & & & \multicolumn{5}{c}{ UK Biobank } \\
& Min & Max & Mean & SD & Min & Max & Mean & SD \\
\hline Age & 18 & 22 & 19.78 & 1.24 & 45 & 78 & 62.66 & 7.38 \\
BMI & 16.30 & 39.15 & 22.29 & 2.83 & 14.94 & 58.04 & 26.60 & 4.419 \\
Early life stress & 25 & 74 & 31.29 & 7.16 & 0 & 20 & 1.68 & 2.32 \\
Depressive symptoms & 0 & 43 & 8.99 & 7.18 & 0 & 27 & 2.45 & 3.39 \\
\hline
\end{tabular}


Figure 1. Mediation model linking genetic risk for higher BMI to higher depressive symptoms, via elevated levels of early life stress 1a. Duke Neurogenetics Study: Discovery sample

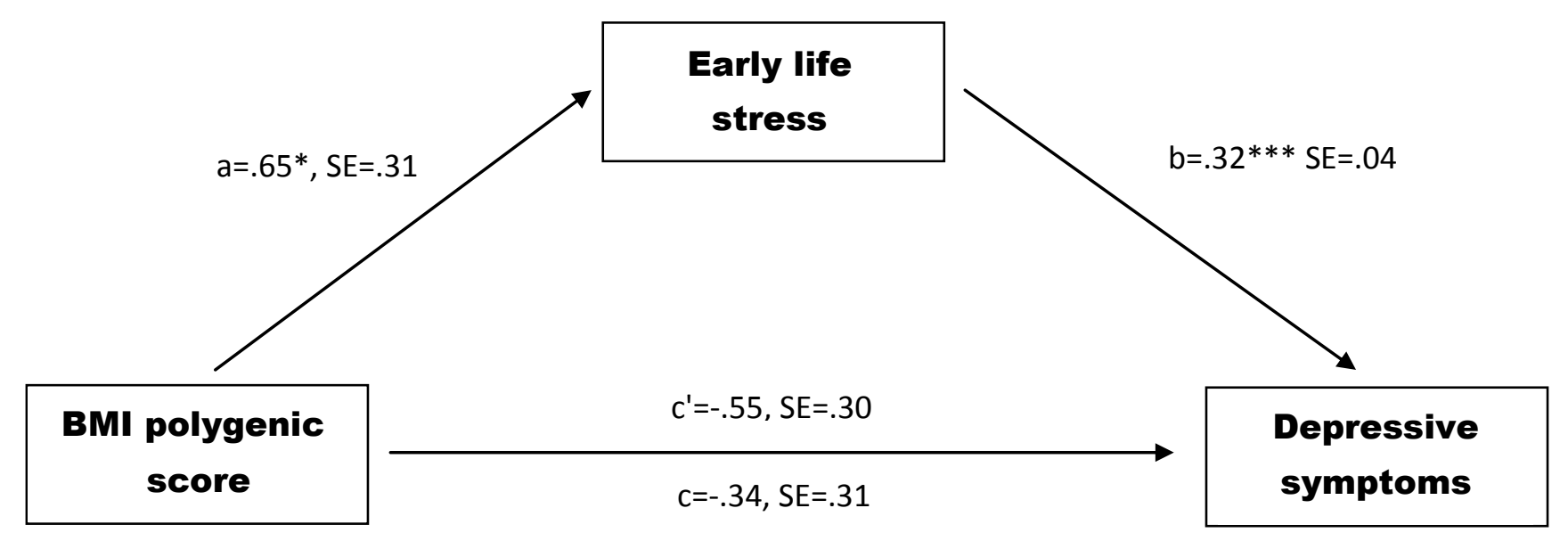


1b. UK Biobank: Replication sample

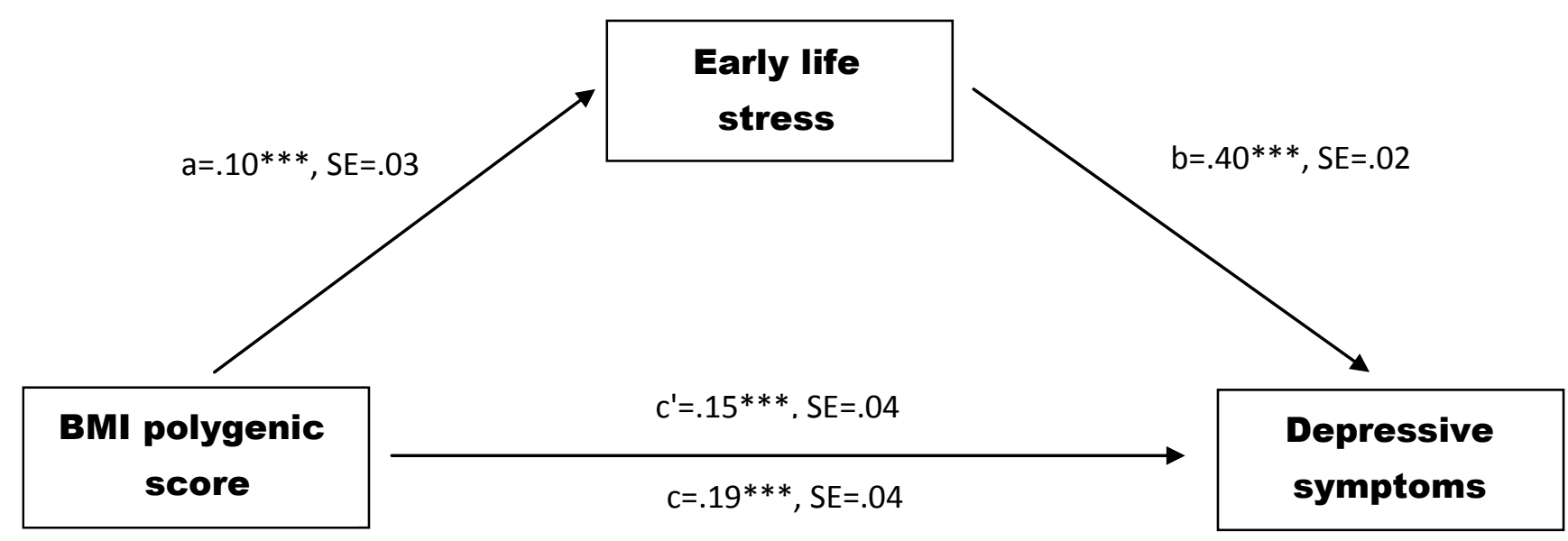

Note. ${ }^{*} p<.05,{ }^{*} p<.01,{ }^{* * *} p<.001 . \mathrm{c}$ - the total effect of the BMI polygenic scores on depressive symptoms; $\mathrm{c}^{\prime}$-the effect of BMI polygenic scores on depressive symptoms, while controlling for ELS. 